\title{
Biomarkers of collagen synthesis predict progression in the PROFILE idiopathic pulmonary fibrosis cohort
}

\author{
Louise A. Organ', Anne-Marie R. Duggan², Eunice Oballa², Sarah C. Taggart², Juliet K. Simpson², \\ Arthur R. Kang'ombe², Rebecca Braybrooke', Philip L. Molyneaux, ${ }^{3,4}$ Bernard North², Yakshitha Karkera², \\ Diana J. Leeming ${ }^{5}$, Morten A. Karsdal ${ }^{5}$, Carmel B. Nanthakumar ${ }^{2}$, William A. Fahy ${ }^{2}$, Richard P. Marshall' \\ R. Gisli Jenkins ${ }^{1,6^{*}}$ and Toby M. Maher ${ }^{3,4^{*}}$ (D)
}

\begin{abstract}
Idiopathic pulmonary fibrosis (IPF) is characterised by excessive extracellular matrix (ECM) deposition and remodelling. Measuring this activity provides an opportunity to develop tools capable of identifying individuals at-risk of progression. Longitudinal change in markers of ECM synthesis was assessed in 145 newly-diagnosed individuals with IPF. Serum levels of collagen synthesis neoepitopes, PRO-C3 and PRO-C6 (collagen type 3 and 6), were elevated in IPF compared with controls at baseline, and progressive disease versus stable disease during follow up, (PRO-C3 $p<0.001$; PRO-C6 $p=0.029$ ). Assessment of rate of change in neoepitope levels from baseline to 3 months (defined as 'slope to month 3': HIGH slope, slope > 0 vs. LOW slope, slope <=0) demonstrated no relationship with mortality for these markers (PRO-C3 (HR 1.62, $p=0.080)$; PINP (HR 0.76, $p=0.309)$; PRO-C6 (HR 1.14, $p=0.628)$ ). As previously reported, rising concentrations of collagen degradation markers C1M, C3M, C6M and CRPM were associated with an increased risk of overall mortality $(\mathrm{HR}=1.84, \mathrm{Cl} 1.03-3.27, p=0.038, \mathrm{HR}=2.44, \mathrm{Cl} 1.39-4.31, p=0.002 ; \mathrm{HR}=2.19, \mathrm{Cl} 1.25-3.82, p=0.006$; $\mathrm{HR}=2.13 \mathrm{Cl} 1.21-3.75, p=0.009$ respectively).

Elevated levels of PRO-C3 and PRO-C6 associate with IPF disease progression. Collagen synthesis and degradation biomarkers have the potential to enhance clinical trials in IPF and may inform prognostic assessment and therapeutic decision making in the clinic.
\end{abstract}

\section{Take home message}

This study demonstrates that synthesis neoepitopes for collagen type 3 and 6 , but not the N-terminal propeptide of collagen type 1 , distinguish individuals with IPF who have progressive disease from those with more stable disease.

\section{Background}

Idiopathic pulmonary fibrosis (IPF) is a fatal condition with a dismal untreated prognosis and despite the approval of two anti-fibrotic therapies remains a major clinical challenge. At present there are no available

\footnotetext{
* Correspondence: gisli.jenkins@nottingham.ac.uk; t.maher@rbht.nhs.uk 'Division of Respiratory Medicine, University of Nottingham, Nottingham, UK ${ }^{3} \mathrm{NIHR}$ Respiratory Clinical Research Facility, Royal Brompton Hospital, London, UK

Full list of author information is available at the end of the article
}

measures for stratifying individuals with IPF to specific therapies nor, importantly, to determine response to therapy. Furthermore, although forced vital capacity $(\mathrm{FVC})$ is accepted as a regulatory end-point in clinical trials, the relative insensitivity of physiological measures to change in fibrosis necessitates studies with a minimum duration of 52 weeks [1]. Therefore, there is an urgent need to identify biomarkers for various aspects of disease behaviour in IPF; particularly those that detect individuals at greater risk of progression and which stratify individuals into better defined endotypes (as a path to enabling effective targeting of therapies to individuals most likely to benefit).

Excessive extracellular matrix (ECM) deposition is central to the pathogenesis of IPF. The production, deposition and remodelling of collagen, a major component of ECM, is a dynamic process [2]. In healthy lung, there is a balance

(C) The Author(s). 2019 Open Access This article is distributed under the terms of the Creative Commons Attribution 4.0 International License (http://creativecommons.org/licenses/by/4.0/), which permits unrestricted use, distribution, and 
between degradation and synthesis of collagen and other ECM proteins [2], which is disturbed in the lungs of individuals with fibrosis [3-5]. During collagen turnover, distinct peptides are generated as by-products of both collagen breakdown and synthesis. The pro-peptide of pro-collagen is cleaved before collagen is incorporated into the matrix. This results in the release of unique fragments for each individual form of collagen [6]. Similarly, during degradation, specific metalloproteases (MMPs) cleave collagen fibres revealing distinct neo-epitopes. Both synthesis peptides and cleavage neo-epitopes are released into the circulation and can be detected in the blood.

We have previously demonstrated, in subjects recruited to the PROFILE (Prospective Observation of Fibrosis in the Lung Clinical Endpoints) study, that serum levels of neoepitopes are higher in individuals with IPF when compared with healthy age-matched controls. Furthermore, we demonstrated that longitudinal changes in serum concentrations of several neo-epitopes track with progression of fibrosis and, by three months, can predict mortality in individuals with IPF [7]. In the current study, we assessed collagen formation markers for their prognostic utility in IPF in a separate cohort of individuals recruited into the PROFILE study. We also compared these synthesis markers to their collagen-equivalent degradation neo-epitopes thus enabling assessment of the relationship between synthesis and degradation during IPF progression.

\section{Methods}

\section{Study design and participants}

As previously described, the PROFILE study is a prospective, multicentre, observational cohort study that recruited individuals with IPF and fibrotic NSIP from two co-ordinating UK centres; Nottingham and Royal Brompton [7, 8]. Participants were enrolled if they had an incident diagnosis confirmed at multidisciplinary discussion according to international consensus guidelines [9]. The cohort of individuals used in the current study represents a sub-group of 145 participants from PROFILE; none of whom were included in the previous PROFILE study on neo-epitopes [7] and all of whom had at least 1 year of follow up at the time neoepitope analyses were performed. A total of 50 physician-verified control participants without history of respiratory disease were recruited from primary care clinics. Samples from 20 of these subjects were used in our earlier study [7]. All participants provided written informed consent. The PROFILE study is registered on ClinicalTrials.gov, numbers NCT01134822 and NCT01110694. For survival analyses, a censoring date of 1st March 2016 was used.

\section{Procedures}

\section{Patient samples}

Serum samples collected from study participants at baseline, 1,3 , and 6 months, and a single serum sample from controls were stored at $-80^{\circ} \mathrm{C}$ until assayed. Studyspecific operating procedures for collection of the serum were used to ensure standardisation across both centres. Briefly, blood was collected with anticoagulant free, serum separation tubes, with coated silica as the clot activator (Becton Dickinson, Winnersh, UK). All samples were processed within $2 \mathrm{~h}$ of collection. Samples were allowed to clot at room temperature for $30 \mathrm{~min}$ and serum was then isolated through centrifugation, and aliquoted before freezing.

\section{Quantification of extracellular matrix related biochemical markers}

Serum samples from patients were analysed using competitive ELISAs for the formation markers of type 1 collagen (P1NP) [10], type 3 collagen (PRO-C3) [11] and type 6 collagen (PRO-C6) [12] as previously described. Additionally, assessment of matrix metalloproteinase (MMP) degraded biglycan (BGM), - type 1 collagen (C1M) [13], -type 3 collagen (C3M) [14], -type 6 collagen (C6M) [15] and C-reactive protein -(CRPM) [16]; was undertaken as previously described [7]. A single aliquot for each subject at each time-point was used for all assays to avoid repeated freeze/thaw of samples. Samples were analysed blinded to the associated clinical data. Performance characteristics of the assays and nonstandard abbreviations are described in the Additional file 1: Table S1.

\section{Follow up}

Lung function measurements for forced vital capacity (FVC) and diffusion capacity for carbon monoxide (DLCO) were performed on participants at baseline, 6 months and 12 months and annually thereafter according to current ATS/ERS guidelines. Percent-predicted values are based on the European Coal and Steel Board reference equations using age and height at the baseline visit [17]. Date of death data was captured from centralised UK National Health Service records.

\section{Statistical analysis}

As previously described, 'progressive disease' was defined as $\geq 10 \%$ decline in FVC and/or death within 12 months. Missing lung function data were not imputed. Where no lung function data beyond baseline was available and the subject had not died within the relevant period, progression status was adjudicated by the responsible physician, after case note review and blinded to biomarker data at the time of adjudication. A total of 9 subjects were adjudicated for progression: 1 subject was adjudicated to have progressed and 8 were adjudicated as stable.

In cases where biomarker data were below the lower limit of detection, values were imputed to be half the lower limit of detection. Values above the upper limit 
were conservatively imputed as the upper limit of detection. Imputation rates ranged between $0.9 \%$ to below $10 \%$ for all markers except C3M that had approximately 19\% imputation. Before statistical analysis all biomarkers were transformed ( $\log _{10}$ transformed where the biomarker is treated as response and $\log _{2}$ where the biomarker is treated as a covariate).

A linear regression model adjusted for gender and age was used to evaluate the association between patient group (healthy controls and IPF subjects) and biomarker values at baseline. Adjusted estimates of group means, 95\% CIs and $p$-values of the estimated mean difference are reported. For serial biomarker data, change from baseline for each participant is represented by a gradient on a linear model where the single explanatory variable is the number of months between the baseline visit and subsequent visits using the recorded visit date. We used a linear mixed-effects model (Mixed Model Repeated Measures (MMRM)) to investigate associations between repeated continuous biomarker response and progression status (progression vs stable), visit, visit by progression status (controls are not included in the model). This analysis was adjusted for age, site, gender and smoking status. Unstructured covariance was assumed for all repeated biomarker response values within a participant. Adjusted estimates of biomarker means (95\% CIs) by progression status at each visit and $p$-values of the test of mean differences between progression vs. stable at each visit are reported. Apart from biomarker data above or below the limits of quantification, all other missing biomarker data was not imputed and was assumed Missing at Random (MAR) implying that after adjusting for important covariates in the model the missing data was independent of the observed data.

Univariate analysis was conducted to examine the effect of baseline biomarker measures on overall survival. Two separate procedures were used; the Kaplan-Meier method for survival curves and a proportional hazards model to derive Hazard Ratios.

A proportional hazards model was used to evaluate association between time to event (overall survival) as response and continuous biomarker value as a covariate. Similarly, in a separate model, baseline \% predicted FVC and separately \% predicted DLco were included as covariates. A proportional hazards model was used to evaluate association between time to event (overall survival) as response and biomarker slope as a factor (defined as 'slope to month 3': HIGH (rising concentrations, slope $>0$ ) versus LOW (stable or falling concentrations, slope $<=0$ ). Hazard ratios represent either risk of mortality for a 2-fold increase in the biomarker value or risk of mortality in participants with rising neoepitope concentrations relative to those with stable or falling concentrations.
Associations between explanatory variables and endpoints were deemed to be significant at the $5 \%$ level. All statistical analyses were conducted with SAS (version 9.3) and were independently quality controlled by a second statistician.

\section{Results}

145 IPF participants recruited in the PROFILE study were assessed in the current analysis. As expected, participants were predominantly male $(81 \%)$ with a mean age of 71.7 years (SD 7.7) and moderate lung function impairment; FVC 79.8 (SD 20.4) \%predicted and DLco 48.2 (SD 17.9) \%predicted. Subject demographics, divided in to those with progressive and stable disease, are presented in Table 1 . The 50 control subjects were $80 \%$ male with a mean age of 64.6 (SD 7.27).

At baseline, levels of PRO-C3 and PRO-C6 but not P1NP, were significantly elevated in individuals with IPF compared with healthy controls (Fig. 1). To enable paired assessment of collagen synthesis and degradation, levels of neoepitopes previously linked with disease progression and survival in the PROFILE study [7] were measured. These included the three degradation neoepitopes which pair with the synthesis markers assessed; C1M (pairs with P1NP), C3M (PRO-C3) and C6M (PRO-C6) and two additional neoepitopes from our previous study, CRPM and BGM. In keeping with our previously presented data, C1M, C3M, C6M and CRPM neoepitopes were significantly elevated in the serum of subjects with IPF compared with controls at baseline (Additional file 1: Figure S1). In subjects with IPF there was mild to moderate correlation between levels of different synthesis neoepitopes (Additional file 1: Table S2). This correlation was strongest for PRO-C3 and PRO-C6 $(\mathrm{r}=0.51)$.

73 subjects had progressive disease whilst 71 had stable disease. Levels of both PRO-C3 and PRO-C6 were higher in individuals with progressive disease compared with those with stable disease at baseline and they remained consistently and significantly higher in individuals with progressive disease at all visits $(1,3$ and 6 months) (Fig. 2). When serial measurements were adjusted for baseline FVC and DLco the numerical differences between progressive and stable subjects remained albeit with a reduction in the significance level at each timepoint (Additional file 1: Figure S3). There were no significant differences between stable and progressive disease at any visit for P1NP (Fig. 2).

Consistent with our previously reported findings, $\mathrm{C} 1 \mathrm{M}, \mathrm{C} 3 \mathrm{M}, \mathrm{C} 6 \mathrm{M}$ and CRPM were also higher in those with progressive disease compared with individuals with stable disease across all time-points beyond baseline (Additional file 1: Figure S2). When values were adjusted for baseline lung function variables this trend remained 
Table 1 Baseline clinical characteristics in participants with idiopathic pulmonary fibrosis. Data are shown for all subjects with IPF and then for the population separated in to those with progressive or stable disease at 12 months. Data are mean (SD) or $n$ (\%), unless otherwise stated. $\mathrm{BMI}=$ body-mass index. FVC = forced vital capacity. DLCO = diffusion capacity for carbon monoxide. $\mathrm{CPI}=$ composite physiological index, *Number of subjects analysed due to missing data

\begin{tabular}{|c|c|c|c|}
\hline Variable & All $(n=145)$ & Progressive $(n=73)$ & Stable $(n=71)$ \\
\hline Age (years) & $71.7(7.7)$ & $71.8(8.3)$ & $71.5(7.1)$ \\
\hline Sex (male) & 117 (80.7\%) & $58(79.5 \%)$ & $59(83.1 \%)$ \\
\hline \multicolumn{4}{|l|}{ Ethnicity } \\
\hline Caucasian & $142(97.9 \%)$ & $71(97.3 \%)$ & $70(98.6 \%)$ \\
\hline Asian & $2(1.4 \%)$ & $1(1.4 \%)$ & $1(1.4 \%)$ \\
\hline Mixed race & $1(0.7 \%)$ & $1(1.4 \%)$ & \\
\hline Ever Smokers & $108(74.5 \%)$ & $54(74.0 \%)$ & $54(76.1 \%)$ \\
\hline $\mathrm{BMI}\left(\mathrm{kg} / \mathrm{m}^{2}\right)$ & $28.4(4.4) * n=144$ & $28.7(4.7){ }^{*} n=72$ & $28.0(4.1){ }^{*} n=71$ \\
\hline FVC (\% predicted) & $79.8(20.4) * n=140$ & $75.6(20.4) * n=69$ & $83.8(19.9){ }^{*} n=70$ \\
\hline DLCO ((\% predicted) & $48.2(17.9) * n=129$ & $41.1(11.9) * n=61$ & $54.7(20.1) * n=67$ \\
\hline 12 month-baseline FVC (\% predicted) & $-4.6(11.7) * n=95$ & $-16.4(10.1) * n=32$ & $1.5(6.9) * n=63$ \\
\hline 12 month-baseline TLCO (\% predicted) & $-3.8(7.7) * n=75$ & $-10.2(6.3) * n=22$ & $-1.1(6.6) * n=53$ \\
\hline $\mathrm{CPI}$ & $44.9(14.9) *_{n}=129$ & $50.5(11.1) * n=61$ & $39.8(16.3){ }^{*} n=67$ \\
\hline Median follow-up days & $1051(584,1180)$ & $609(196,1092)$ & $1128.0(1050,1212)$ \\
\hline
\end{tabular}

albeit with reduced statistical significance (see Figs. S3 and S4 in the Additional file 1).

When comparing ratios of synthesis and degradation markers P1NP:C1M was significantly lower in participants with progressive disease from 1 month onwards compared with stable disease, whilst PROC3:C3M was significantly lower in participants with stable disease at each time point compared with progressive disease (Fig. 3). There were no significant differences between stable and progressive disease at any timepoint for the ratio of PRO-C6:C6M (Fig. 3).

In an unadjusted univariate analysis, baseline concentrations of PRO-C3 (H.R. $1.49(1.04-2.13), p=0.030)$ and P1NP:C1M ratio (H.R. 0.67 (0.53-0.85), $p=0.001)$

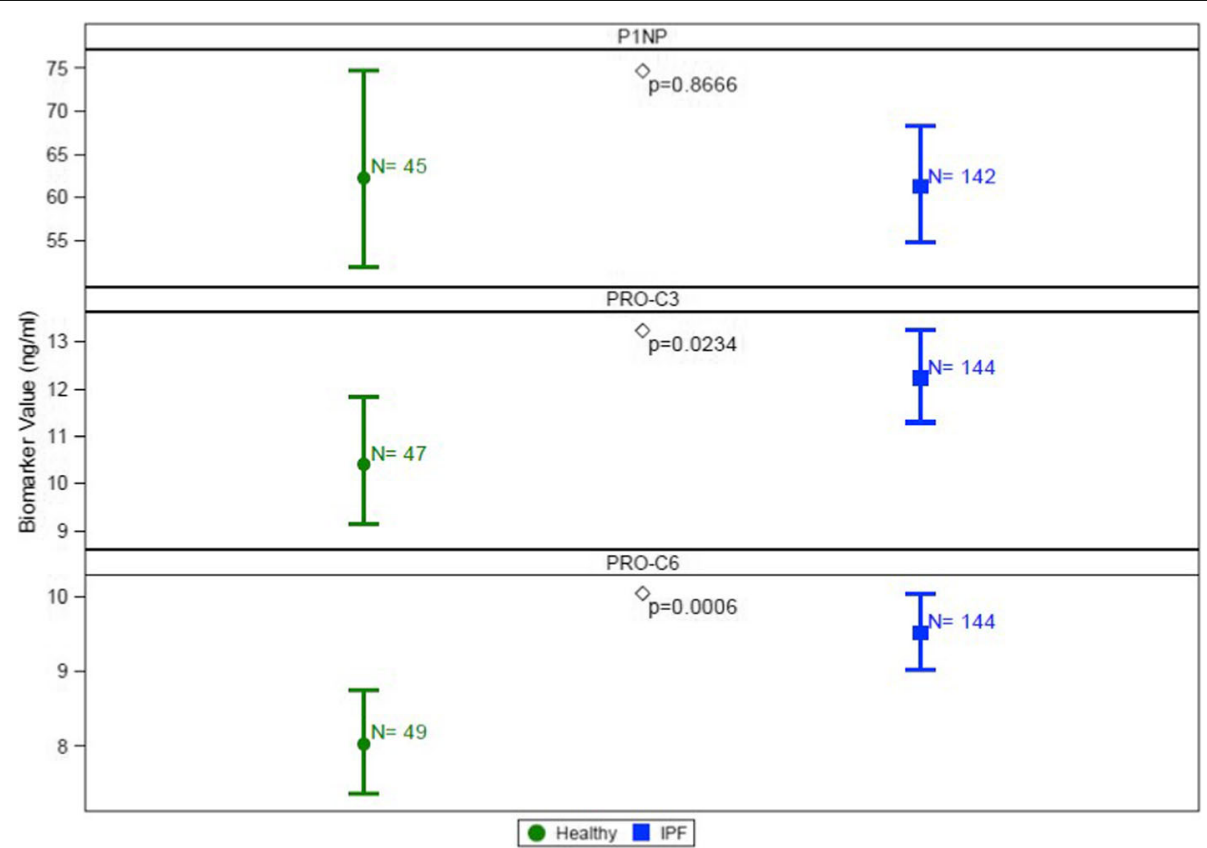

Fig. 1 Baseline comparison of collagen synthesis neoepitope concentrations for collagen-type 1 (P1NP), -type 3 (PRO-C3) and -type 6 (PRO-C6) in healthy controls and participants with idiopathic pulmonary fibrosis. Plots represent mean and $95 \% \mathrm{Cl}$ (error bars) adjusted for age and gender 

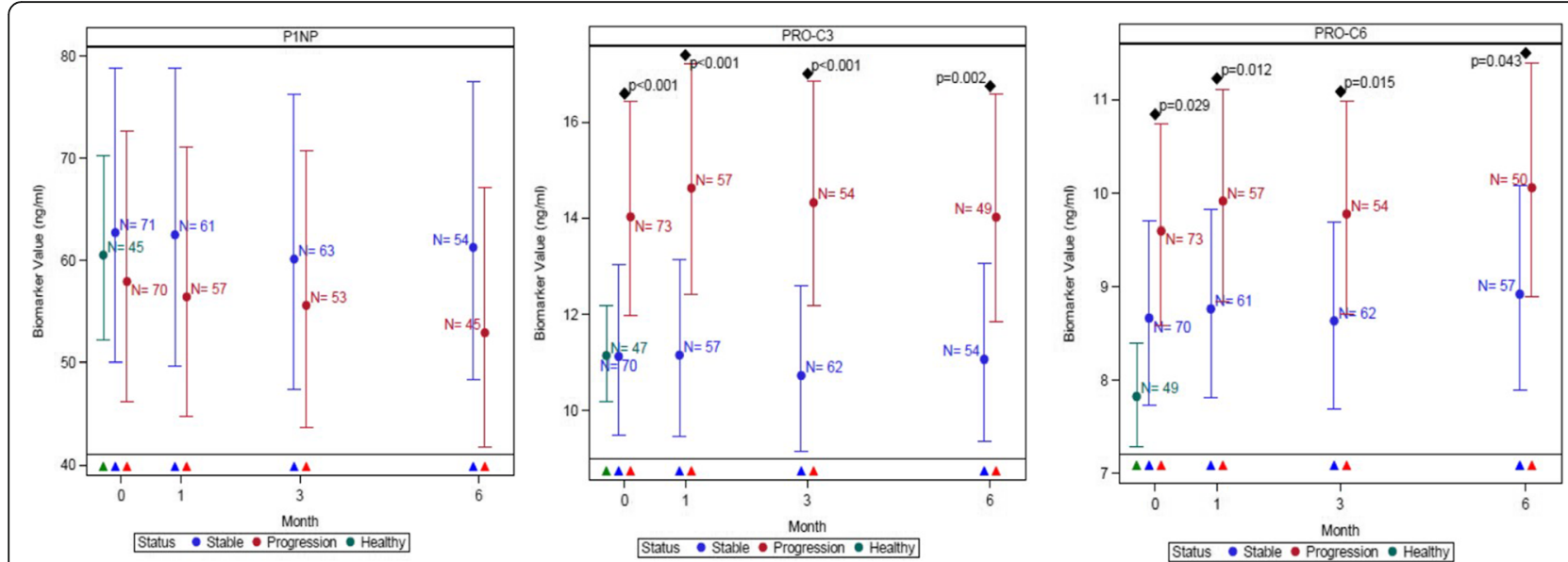

Fig. 2 Comparisons of neoepitope concentrations for collagen synthesis markers in healthy control and participants with stable and progressive IPF, measured at baseline, 1, 3 and 6 months after baseline for collagen-type 1 (P1NP), -type 3 (PRO-C3) and -type 6 (PRO-C6). Plots represent mean and $95 \% \mathrm{Cl}$ (error bars) adjusted for age, sex, site and smoking status. Disease progression was defined as all-cause mortality or $\geqq 10 \%$ decline in forced vital capacity at 12 months. The number of evaluable samples available for analysis at each time point are provided in the graph. $P$ values are provided where significant $(p<0.05)$ differences were observed between stable and progressive disease at a particular time point. Triangles represent which groups were compared at each time point on the graph, based on colour

were predictive of survival (Table 2). As expected, \% predicted FVC at baseline (H.R. $0.22(0.12-0.39, p<0.0001)$ and \% predicted DLco at baseline (H.R. $0.16(0.09-0.30)$, $\mathrm{p}<0.0001$ ) were strongly predictive of survival (Table 2.). Following adjustment for baseline FVC and DLco, P1NP:C1M ratio remained predictive of survival (H.R. 0.77 (0.6-0.99), $p=0.0389)$.

Change over time of the three synthesis markers failed to associate with overall survival (Fig. 4). By contrast, and in keeping with our previously published observations, individuals with rising levels of CRPM, C1M, C3M and $\mathrm{C6M}$ were at high risk of death compared with those with falling/stable levels (HIGH vs LOW) of CRPM (HR 2.13 [95\% CI 1.21-3.75] $p=0.009$, C1M (HR 1.84 [CI 95\% 1.03-3.27] $P=0.038$ ), C3M (HR 2.44 [CI 95\% 1.39-4.31] $p=0.002)$ and C6M (HR 2.19 [1.253.82], $p=0.006$ ) (Fig. 4). Rising levels of the ratio of PRO-C6:C6M were associated with lower mortality (HR $0.55[0.32-0.95], p=0.032$ ), however, no relationship was observed with levels of P1NP:C1M or PRO-C3:C3M (Fig. 4). Following correction for baseline lung function the association between change in $\mathrm{C} 1 \mathrm{M}$ and PRO-C6:
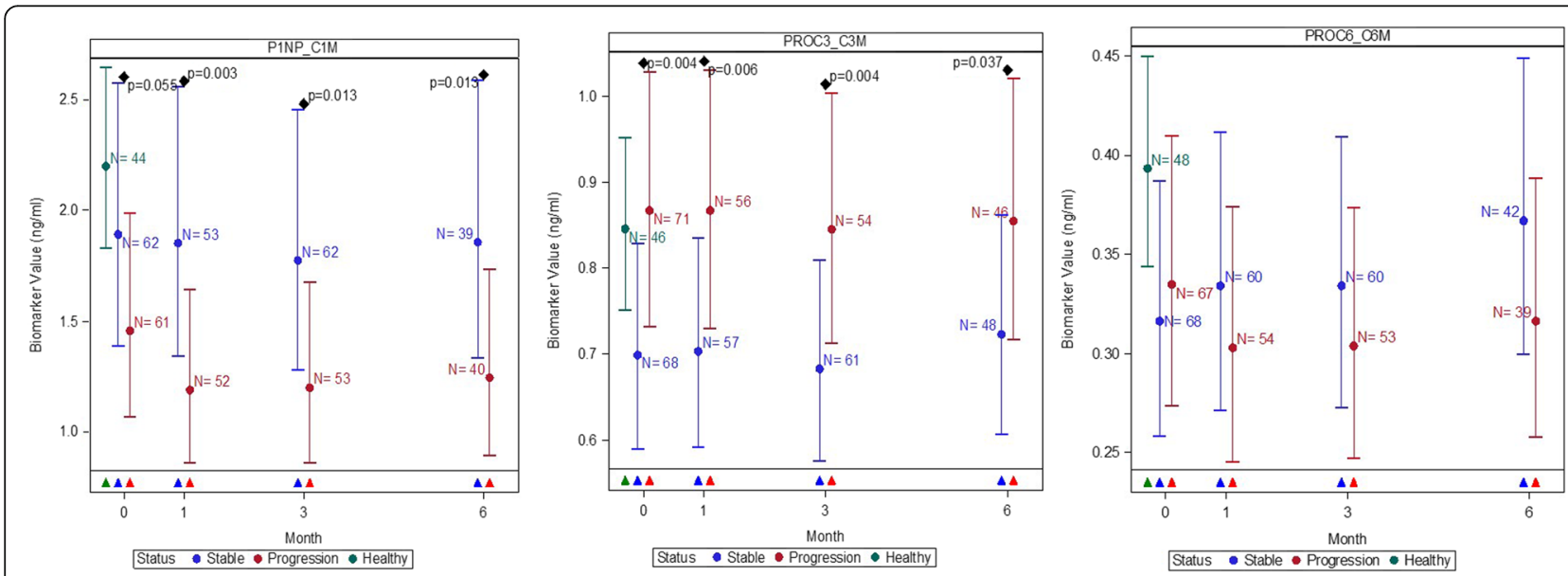

Fig. 3 Comparisons of absolute values in the ratio of neoepitope collagen synthesis to degradation markers in healthy controls and participants with stable and progressive IPF, measured at baseline, 1, 3 and 6 months for: collagen-type 1 (P1NP:C1M), -type 3 (PRO-C3:C3M) and -type 6 (PRO-C6:C6M). Plots represent mean and 95\% Cl (error bars) adjusted for age, sex, site and smoking status. Disease progression was defined as allcause mortality or $\geqq 10 \%$ decline in forced vital capacity at 12 months. The number of evaluable samples available for analysis at each time point are provided in the graph. $P$ values are provided where significant $(p<0.05)$ differences were observed between stable and progressive disease at a particular time point. Triangles represent which groups were compared at each time point on the graph, based on colour 
Table 2 Unadjusted and adjusted (for baseline FVC and DLCO) univariate analysis of overall survival in relation to baseline measures of collagen synthesis neoepitopes. Data shown for collagen-type 1 (P1NP), -type 3 (PRO-C3) and -type 6 (PRO-C6), the ratio of collagen synthesis: degradation ratios for collagen-type 1 (P1NP:C1M), -type 3 (PRO-C3: C3M), and -type 6 (PRO-C6: C6M) and lung function measurements (\% predicted FVC and \% predicted DLCO) in IPF subjects. The Hazard Ratio is based on a two-fold increase in the variable. Data are expressed as mean hazard ratio (HR) with 95\% Cls for all thresholds and represent the associated change in mortality risk based on a 2 -fold increase in the explanatory value

\begin{tabular}{|c|c|c|c|c|c|}
\hline \multirow[b]{2}{*}{ Group } & \multirow[b]{2}{*}{ Variable } & \multicolumn{2}{|l|}{ Univariate } & \multicolumn{2}{|l|}{ Adjusted for LF } \\
\hline & & Hazard Ratio $(95 \% \mathrm{Cl})$ & $P$ value & Hazard Ratio $(95 \% \mathrm{Cl})$ & $P$ value \\
\hline & P1NP & $0.79(0.60,1.05)$ & 0.1013 & $0.81(0.60,1.11)$ & 0.1871 \\
\hline & PRO-C3 & $1.49(1.04,2.13)$ & 0.0295 & $1.20(0.74,1.93)$ & 0.4577 \\
\hline & PRO-C6 & $1.47(0.84,2.59)$ & 0.1787 & $1.11(0.57,2.16)$ & 0.7600 \\
\hline & P1NP:C1M & $0.67(0.53,0.85)$ & 0.0011 & $0.77(0.60,0.99)$ & 0.0389 \\
\hline & PROC3:C3M & $1.30(0.91,1.85)$ & 0.1542 & $1.17(0.77,1.79)$ & 0.4679 \\
\hline & PROC6:C6M & $0.87(0.63,1.21)$ & 0.4162 & $0.86(0.59,1.26)$ & 0.4319 \\
\hline & $\%$ pred FVC & $0.22(0.12,0.39)$ & $<0.0001$ & & \\
\hline & $\%$ pred TLCO & $0.16(0.09,0.30)$ & $<0.0001$ & & \\
\hline
\end{tabular}

C6M no longer remained significant (Additional file 1: Table S3). There was moderate correlation between changes in different neoepitopes (Additional file 1: Table S4).

\section{Discussion}

Remodelling of extracellular matrix is pivotal to the development and progression of IPF and results in distinct alterations in collagen turnover. In the current study, we measured synthesis markers of three collagens important in the development of IPF; namely collagen type- 1 (P1NP), - 3 (PRO-C3) and - 6 (PRO-C6) $[4,18,19]$. Given that fibrogenesis in IPF likely reflects an imbalance between synthesis and degradation of collagen, we also measured paired markers of degradation for the same collagens: type- 1 (C1M), -3 (C3M) and -6 (C6M). For comparison with our prior observations [7], we also measured degradation of the matrix molecule biglycan (BGM) and $C$ reactive protein (CRPM). Our data demonstrate that synthesis markers for type-3 (PRO-C3) and-6 (PRO-C6) collagen are elevated in subjects with IPF compared with healthy controls and distinguish individuals with progressive disease, suggesting that these biomarkers may reflect underlying pathophysiology driving disease activity in IPF.

Collagen type-3, a fibrillar collagen, forms a major component of the lung interstitial extracellular matrix and is synthesised by activated fibroblasts [20]. Our findings support earlier studies demonstrating that synthesis of collagen type- 3 is increased in IPF, with high levels of type-3 pro-collagen peptide reported in serum [18] and lavage fluid $[21,22]$. In the case of collagen type-3, the synthesis neoepitope, PRO-C3, appears to be a better biomarker of progressive disease than the paired degradation biomarker, C3M. Interestingly, in subjects with more stable disease, the ratio of $\mathrm{PRO}-\mathrm{C} 3$ to $\mathrm{C} 3 \mathrm{M}$ is significantly lower; supporting the idea that synthesis, and increased turnover in general, may be reflective of active remodelling and disease progression.

Alongside collagen type-3, our data suggest that changes in collagen type- 6 are also informative of disease behaviour in IPF and may be an important driver of fibrosis. Collagen type- 6 filaments are an integral component found at the interface between the basement membrane and interstitial matrix of the lung, and act as an important network for attachment of basement membranes, collagen fibres and cells. Our results support the importance of this collagen in the development of fibrosis. Previous research suggests that collagen type- 6 fragments have signalling properties during wound healing and fibrogenesis and may drive poorer outcomes in fibrotic diseases [23]. Compared with other collagens, type- 6 collagen has been shown to be a potent inducer of the differentiation and activation of fibroblasts to highly synthetic myofibroblasts; a key pathway leading to the production of collagen and ECM proteins [24]. In addition, increased levels of serum collagen type- 6 markers have been demonstrated to act as an early predictor of fibrosis in the liver [15]. This evidence, coupled with data from the current study, suggest that collagen type- 6 may in itself be a driver of the fibrotic process, which in turn serves as a useful marker of IPF progression.

Intriguingly, whilst increases in collagen type-1 production are associated with TGF $\beta$ - activation of IPF fibroblasts [25], and have been observed in IPF lung [26], we did not find elevated levels of collagen type 1 synthesis neoeptiopes in the serum of IPF subjects. However, collagen type- 1 is also the predominant collagen found in bone and it has been estimated that $90 \%$ of the 

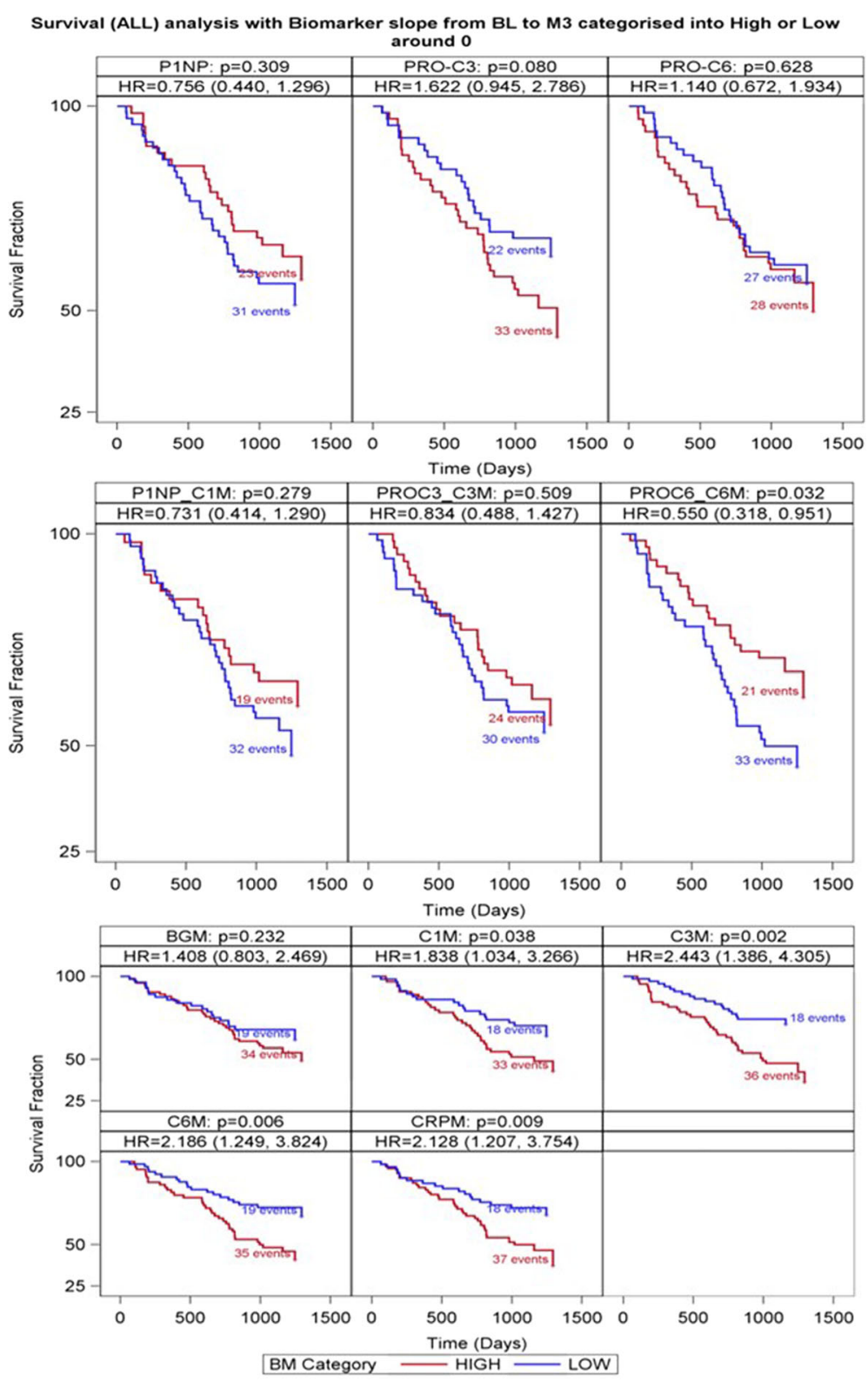

HIGH = Rising biomarker category: participants with rising 3 month
change in neoepitope concentration $0 \geq \mathrm{ng} / \mathrm{mL}$ per month
LOW $=$ Stable biomarker category: participants with stable or falling 3
month change in neoepitope concentration $<0 \mathrm{ng} / \mathrm{mL}$ per month

Fig. 4 Effect of 3 month change in neoepitope concentrations on overall survival. Subjects with IPF were dichotomised into HIGH or LOW groups, to determine the effect of 3-month change in neoepitope concentration on overall survival, where: $\mathrm{HIGH}=$ rising $(0>\mathrm{ng} / \mathrm{mL}$ per month) neoepitope concentrations from baseline to month 3, >or LOW = stable or falling $(\leq 0 \mathrm{ng} / \mathrm{mL}$ per month) neoepitope concentration from baseline to month 3 . For ratio values, the slope values were derived following the creation of the ratio variables and subjects were dichotomised into HGH or LOW slope groups. Hazard ratio represents the mortality risk in participants with rising neoepitope concentrations relative to those with stable or falling concentrations. Data shown in: top panel - collagen synthesis markers P1NP, PRO-C3, PRO-C6; mid panel - ratio of collagen synthesis/degradation markers: P1NP:C1M, PRO-C3:C3M and PRO-C6:C6M; bottom panel -BGM, C1M, C3M, C6M, CRPM). The number of deaths in each group is shown for each biomarker. P values presented relate to the comparison of the Kaplan-Meir sunvival curves for each dichotomised measure

synthesis of this collagen measurable in serum is bone derived [27]. This may explain why there was no difference observed in P1NP, the measured marker of collagen type-1 synthesis, between IPF subjects and health controls and why differences were not seen between stable and progressive disease. In contrast to P1NP, the serum 
degradation marker of collagen type-1, C1M, does not measure bone-derived degradation as the epitope is denatured in bone by osteoclast-released cathepsin $\mathrm{K}$ [13]. This disparity in the pools of collagen type- 1 being measured may account for the differences observed between this pair of synthesis and degradation neoepitopes.

Consistent with our previous study, baseline levels of $\mathrm{C} 1 \mathrm{M}, \mathrm{C} 3 \mathrm{M}, \mathrm{C6M}$ and CRPM were elevated in subjects with IPF compared with healthy controls. Furthermore, consistent with our prior observations, elevated CRPM distinguished progressive from stable disease from as early as one month after recruitment. In our previous study BGM, C1M, C3M and C6M were also different between individuals with stable and progressive IPF, but these differences only became significant at 6 months. Interestingly, in the current study, the levels of $\mathrm{C} 1 \mathrm{M}$ and C6M were significantly higher from 1 month onwards.

Rising levels over 3 months of matrix synthesis markers were not predictive of mortality. This is in distinct contrast with changing levels of the degradation markers CRPM, C1M, C3M and C6M; for each of these markers rising levels over 3 months identified subjects with an increased risk of death. The predictive value of these markers, as shown by the hazard ratios, was consistent with previous results [7]. A changing ratio over 3 months of synthesis and degradation markers for type 6 collagen, PRO-C6:C6M, was also predictive of mortality albeit less strongly than C6M alone especially after adjustment for baseline lung function. It is possible that the different characteristics of the synthesis and degradation markers may reflect the specific location of individual collagens within the fibrotic lung and their susceptibility to degradation as fibrosis progresses. Alternatively, as is likely to be the case for P1NP, other anatomical pools of collagen turnover, including bone [27] and liver [15], may dilute the serum signal for individual neoepitopes thus limiting their suitability as markers of IPF severity or disease progression.

The absence of reliable and validated short-term measurements that predict disease progression, survival and response to treatment represent a major obstacle in the management of IPF. Physiological measurements, particularly FVC, remain the primary method for monitoring IPF disease progression in both registration clinical trials and clinical practice $[28,29]$. Whilst changes in lung function are likely to remain a gold standard for assessing IPF progression, there are several short-comings of these measurements. Firstly, they are relatively insensitive to subtle changes in disease severity necessitating measurement over longer timeframes (52 weeks in clinical trials) before disease progression can be reliably determined. Although a recent study has demonstrated that daily home spirometry is predictive of disease progression within 3 months, there are some potential limitations with this method, particularly long-term compliance [30]. Secondly, current treatments only slow decline in FVC making determination of treatment response in individuals almost impossible. Serum biomarkers, such as those measured in the current study, have the potential to overcome some of these challenges, by providing a minimally invasive, short-term readout that could supplement FVC measurements to give a more reliable assessment of disease progression and response to anti-fibrotic therapy.

We have shown that neoepitopes reflecting collagen synthesis and degradation in IPF are informative of prognosis, are able to distinguish progressive from non-progressive disease and provide a 3-month read out of change in disease severity. The utility of these observations is currently being tested in a randomised placebo control trial of nintedanib in individuals with IPF and preserved lung function (NCT02788474) [31]. If neo-epitopes can be shown to change with therapy, then these markers may be of use in shortening future clinical trials and for determining response to therapy in the clinic.

A major strength of our study is the prospective longitudinal design, which enabled blood sampling to be standardised and enabled collection of robust outcome data. Furthermore, the PROFILE cohort is representative of real-world patients with IPF and includes many subjects who would not have been enrolled in clinical trials [32]. As such our findings are more translatable to clinical practice than insights derived from clinical trial cohorts. Furthermore, recruitment of the PROFILE cohort commenced before effective therapy for IPF became available. As such, none of the cohort described in this manuscript were on antifibrotic therapy during the first 12 months of follow up.

The major weakness of this current study is that we undertook analysis of synthesis neoepitopes in a single cohort and did not adhere to a discovery-validation design. However, as PROFILE is the only longitudinal, realworld study that includes treatment-naive patients with multiple biological samples, and without the limitations posed by clinical trial cohorts, that we are aware of, external replication of this study is not currently possible. We feel that this limitation is partially offset by our demonstration that degradation neo-epitopes behave consistently with our previous observations, which were performed in discovery, and validation sample sets. As such we believe that this confirmation of the usefulness of collagen degradation biomarkers provides indirect validation of our findings and supports our conclusions regarding the potential utility of collagen synthesis biomarkers. However, we hope that others will, in future be able to validate the relationships which we have observed between synthesis biomarkers and disease activity and confirm the relevance of ratios of paired collagen synthesis and degradation markers. 


\section{Conclusion}

This study demonstrates that collagen type- 3 and -6 synthesis biomarkers are elevated in individuals with IPF compared with controls and differ in levels between stable and progressive disease. In addition, this study re-confirms the value of a panel of ECM degradation biomarkers that are predictive of disease progression and survival in IPF. This panel of biomarkers provides informative insights into matrix turnover and underlying pathophysiology driving disease progression, which may assist in the development of precision approaches to IPF treatment. Furthermore, the predictive prognostic value of change in these biomarkers is discernible within 3 months, providing much needed short-term measurements to assist the identification of at-risk IPF patients, which could be used to supplement physiological assessment to allow for better disease management through targeted and earlier therapeutic intervention strategies. Further validation of these findings is required in additional real-world cohorts of individuals with IPF and other fibrosing lung diseases.

\section{Additional file}

Additional file 1: Table S1. Target and assay performance characteristics for the ELISA assays specific to each neoepitope. Table S2. Spearman correlations for baseline serum concentrations of synthesis neoepitopes in control and IPF subjects. Table S3. Unadjusted and adjusted (for baseline FVC and DLCO) univariate analysis of overall survival in relation to 3-month change in neoepitope concentrations. Table S4. Spearman correlations for 3-month change in serum concentrations of synthesis and degradation neoepitopes in IPF subjects. Figure S1. Baseline comparison of collagen degradation neoepitope (BGM, C1M, C3M, C6M and CRPM) concentrations in healthy controls $(n=50)$ and participants with idiopathic pulmonary fibrosis $(n=145)$. Plots represent mean and $95 \% \mathrm{Cl}$ (error bars) adjusted for gender. Figure S2. Comparison of neoepitope concentrations in healthy controls (green) and participants with stable (blue) and progressive (red) idiopathic pulmonary fibrosis at baseline and subsequently at 1-, 3- and 6months. Figure S3. Comparison of synthesis neoepitope concentrations adjusted for baseline FVC and DLCo \%predicted in healthy controls (green) and participants with stable (blue) and progressive (red) idiopathic pulmonary fibrosis at baseline and subsequently at 1-, 3- and 6-months. Figure S4. Comparison of degradation neoepitope concentrations adjusted for baseline FVC and DLco \%predicted in healthy controls (green) and participants with stable (blue) and progressive (red) idiopathic pulmonary fibrosis at baseline and subsequently at 1-, 3- and 6-months. Figure S5. Comparison of neoepitope concentrations adjusted for baseline FVC and DLCo \%predicted in healthy controls (green) and participants with stable (blue) and progressive (red) idiopathic pulmonary fibrosis at baseline and subsequently at 1-, 3- and 6-months. (DOCX $1380 \mathrm{~kb}$ )

\footnotetext{
Abbreviations

BGM: Biglcan degraded by matrix metalloprotease; BMI: Body mass index; C1M: Collage type 1 degraded by matrix metalloprotease; C3M: Collagen type 3 degraded by matrix metalloprotease; C6M: Collagen type 6 degraded by matrix metalloprotease; CPI: composite physiological index; CRPM: Creactive protein degraded by matrix metalloprotease; DLCO: diffusion capacity for carbon monoxide; ECM: Extra cellular matrix; FVC: Forced vital capacity; IPF: Idiopathic pulmonary fibrosis; MMP: Matrix metalloprotease; MMRM: Mixed Model Repeated Measures; P1NP: collagen synthesis neoepitopes for collagen-type 1; PRO-C3: collagen synthesis neoepitopes for collagen-type 3; PRO-C6: collagen synthesis neoepitopes for collagen-type 6; PROFILE: Prospective Observation of Fibrosis in the Lung Clinical Endpoints; TGFB: Transforming growth factor beta
}

\section{Acknowledgements}

Not applicable.

\section{Authors' contributions}

RGJ, TMM, RPM, WAF, JKS, EO conceived and designed the study; RB, PLM and participated in recruitment of study patients and collected their data. DJL and MAK developed and undertook the neoepitope assays. EO, AMD, ST, ARK, CBN, LO, WAF, RGJ, and TMM undertook the data analysis. L. O, T. M prepared manuscript. All authors agree with manuscript results and conclusions, made critical revisions and approved final version.

\section{Funding}

The PROFILE study was funded by the Medical Research Council (G0901226) and GlaxoSmithKline R\&D (CRT114316) and was sponsored by Nottingham University and Royal Brompton and Harefield NHS Foundation Trust. T.M. Maher is supported by an NIHR Clinician Scientist Fellowship (NIHR Ref: CS2013-13-017) and a British Lung Foundation Chair in Respiratory Research (C17-3).

\section{Availability of data and materials}

Data is available on reasonable written request to the PROFILE study steering committee.

\section{Ethics approval and consent to participate}

The PROFILE study is registered on clinicaltrials.gov (NCT01134822 and NCT01110694). The study was approved by the Royal Free Hospital Research Ethics Committee Ref 10/H0720/12.

\section{Consent for publication}

All subjects provided signed informed consent including for publication of all results arising from the PROFILE study.

\section{Competing interests}

WAF, JKS, EO, CBN, ARK are employees and shareholders of GSK. ST, BN, YK and AMD are working on behalf of GSK. TMM has, via his institution, received industry-academic funding from GlaxoSmithKline R\&D and UCB and has received consultancy or speaker fees from Apellis, Astra Zeneca, Bayer, Biogen Idec, Boehringer Ingelheim, Galapagos, GlaxoSmithKline R\&D, Indalo, Pliant, ProMetic, Roche, Samumed and UCB. RGJ has received industryacademic funding from GlaxoSmithKline R\&D, Novartis and Biogen Idec and has received consultancy or speaker fees from InterMune, Medlmmune, Pulmatrix, Boehringer Ingelheim, and GlaxoSmithKline R\&D. MAK and DJL are employees and shareholders of Nordic Biosciences and hold patents relating to neoepitopes.

\section{Author details}

${ }^{1}$ Division of Respiratory Medicine, University of Nottingham, Nottingham, UK. ${ }^{2}$ Fibrosis Discovery Performance Unit, GlaxoSmithKline R\&D, GlaxoSmithKline Medicines Research Centre, Gunnels Wood Road, Stevenage SG1 2NY, UK. ${ }^{3} \mathrm{NIHR}$ Respiratory Clinical Research Facility, Royal Brompton Hospital, London, UK. ${ }^{4}$ National Heart and Lung Institute, Imperial College, Sir Alexander Fleming Building, London SW7 2AZ, UK. ${ }^{5}$ Nordic Bioscience A/S, Biomarkers and Research, Herlev Hovedgade 205-207, DK-2730 Herlev, Denmark. ${ }^{6}$ Respiratory Research Unit, Nottingham University Hospitals NHS Trust, Nottingham NG5 1PB, United Kingdom.

Received: 6 February 2019 Accepted: 1 July 2019

Published online: 12 July 2019

References

1. Rose D, Montgomery A. Idiopathic pulmonary fibrosis: clinically meaningful primary endpoints in phase 3 clinical trials. Am J Respir Crit Care Med. 2013; 187:1269.

2. Hynes RO. The extracellular matrix: not just pretty fibrils. Science. 2009;326: 1216-9.

3. Selman M, Montano M, Ramos C, Chapela R. Concentration, biosynthesis and degradation of collagen in idiopathic pulmonary fibrosis. Thorax. 1986; 41:355-9.

4. Kulkarni T, O'Reilly P, Antony VB, Gaggar A, Thannickal VJ. Matrix remodeling in pulmonary Fibrosis and emphysema. Ja: American journal of respiratory cell and molecular biology; 2016. 
5. Leeming DJ, Sand JM, Nielsen MJ, Genovese F, Martinez FJ, Hogaboam CM, Han MK, Klickstein LB, Karsdal MA. Serological investigation of the collagen degradation profile of patients with chronic obstructive pulmonary disease or idiopathic pulmonary fibrosis. Biomarker insights 2012; 7: BMI. S9415.

6. McKleroy W, Lee T-H, Atabai K. Always cleave up your mess: targeting collagen degradation to treat tissue fibrosis. Am J Phys Lung Cell Mol Phys. 2013;304:L709-21.

7. Jenkins RG, Simpson JK, Saini G, Bentley JH, Russell A-M, Braybrooke R, Molyneaux PL, McKeever TM, Wells AU, Flynn A. Longitudinal change in collagen degradation biomarkers in idiopathic pulmonary fibrosis: an analysis from the prospective, multicentre PROFILE study. Lancet Respir Med. 2015:3:462-72.

8. Maher TM, Oballa E, Simpson JK, Porte J, Habgood A, Fahy WA, Flynn A, Molyneaux PL, Braybrooke R, Divyateja H. An epithelial biomarker signature for idiopathic pulmonary fibrosis: an analysis from the multicentre PROFILE cohort study. Lancet Respir Med. 2017;5:946-55.

9. Raghu G, Collard HR, Egan JJ, Martinez FJ, Behr J, Brown KK, Colby TV, Cordier J-FF, Flaherty KR, Lasky JA, Lynch DA, Ryu JH, Swigris JJ, Wells AU, Ancochea J, Bouros D, Carvalho C, Costabel U, Ebina M, Hansell DM, Johkoh T, Kim DS, King TE, Kondoh Y, Myers J, Müller NL, Nicholson AG, Richeldi L, Selman M, Dudden RF, Griss BS, Protzko SL, Schünemann HJ, Fibrosis AEJACIP. An official ATS/ERS/JRS/ALAT statement: idiopathic pulmonary fibrosis: evidence-based guidelines for diagnosis and management. Am J Respir Crit Care Med. 2011;183:788-824.

10. Leeming DJ, Larsen D, Zhang C, Hi Y, Veidal S, Nielsen R, Henriksen K, Zheng Q, Barkholt V, Riis B. Enzyme-linked immunosorbent serum assays (ELISAs) for rat and human N-terminal pro-peptide of collagen type I (PINP) —assessment of corresponding epitopes. Clin Biochem. 2010;43:1249-56.

11. Nielsen MJ, Nedergaard AF, Sun S, Veidal SS, Larsen L, Zheng Q, Suetta C, Henriksen K, Christiansen C, Karsdal MA. The neo-epitope specific PRO-C3 ELISA measures true formation of type III collagen associated with liver and muscle parameters. Am J Transl Res. 2013;5:303.

12. Nedergaard A, Sun S, Karsdal MA, Henriksen K, Kjær M, Lou Y, He Y, Zheng Q, Suetta C. Type VI collagen turnover-related peptides-novel serological biomarkers of muscle mass and anabolic response to loading in young men. J Cachexia Sarcopenia Muscle. 2013;4:267-75.

13. Leeming DJ, He Y, Veidal S, Nguyen Q, Larsen D, Koizumi M, SegoviaSilvestre T, Zhang C, Zheng Q, Sun S. A novel marker for assessment of liver matrix remodeling: an enzyme-linked immunosorbent assay (ELISA) detecting a MMP generated type I collagen neo-epitope (C1M). Biomarkers. 2011;16:616-28.

14. Barascuk N, Veidal S, Larsen L, Larsen D, Larsen M, Wang J, Zheng Q, Xing R, Cao Y, Rasmussen LM. A novel assay for extracellular matrix remodeling associated with liver fibrosis: an enzyme-linked immunosorbent assay (ELISA) for a MMP-9 proteolytically revealed neo-epitope of type III collagen. Clin Biochem. 2010:43:899-904.

15. Veidal SS, Karsdal MA, Vassiliadis E, Nawrocki A, Larsen MR, Nguyen QHT, Hägglund P, Luo Y, Zheng Q, Vainer B. MMP mediated degradation of type $\mathrm{VI}$ collagen is highly associated with liver fibrosis-identification and validation of a novel biochemical marker assay. PLoS One. 2011;6:e24753.

16. Skjøt-Arkil H, Schett G, Zhang C, Larsen DV, Wang Y, Zheng Q, Larsen MR, Nawrocki A, Bay-Jensen A-C, Henriksen K. Investigation of two novel biochemical markers of inflammation, matrix metalloproteinase and cathepsin generated fragments of $C$-reactive protein, in patients with ankylosing spondylitis. Clin Exp Rheumatol. 2012;30:371-9.

17. Cotes J, Chinn D, Quanjer PH, Roca J, Yernault J-C. Standardization of the measurement of transfer factor (diffusing capacity). Eur Respiratory Soc. 1993.

18. Kirk J, Bateman E, Haslam P, Laurent GJ, Turner-Warwick M. Serum type III procollagen peptide concentration in cryptogenic fibrosing alveolitis and its clinical relevance. Thorax. 1984;39:726-32.

19. Specks U, Nerlich A, Colby TV, Wiest I, Timpl R. Increased expression of type VI collagen in lung fibrosis. Am J Respir Crit Care Med. 1995;151:1956-64.

20. Frantz C, Stewart KM, Weaver VM. The extracellular matrix at a glance. J Cell Sci 2010; 123: 4195-4200.

21. Low RB, Giancola MS, King T, Chapitis J, Vacek P, Davis GS. Serum and bronchoalveolar lavage n-terminal type III procollagen peptides in idiopathic pulmonary fibrosis. Am Rev Respir Dis 1992; 146: 701-701.

22. Bjermer L, Lundgren R, Hällgren R. Hyaluronan and type III procollagen peptide concentrations in bronchoalveolar lavage fluid in idiopathic pulmonary fibrosis. Thorax. 1989;44:126-31.
23. Karsdal M, Nielsen $S$, Leeming $D$, Langholm L, Nielsen M, Manon-Jensen $T$, Siebuhr A, Gudmann N, Rønnow S, Sand J. The good and the bad collagens of fibrosis-their role in signaling and organ function. Adv Drug Deliv Rev. 2017:121:43-56.

24. Naugle JE, Olson ER, Zhang X, Mase SE, Pilati CF, Maron MB, Folkesson HG, Horne WI, Doane KJ, Meszaros JG. Type VI collagen induces cardiac myofibroblast differentiation: implications for postinfarction remodeling. Am J Phys Heart Circ Phys. 2006;290:H323-30.

25. Ramos C, Montaño M, García-Alvarez J. Fibroblasts from idiopathic pulmonary fibrosis and normal lungs differ in growth rate, apoptosis, and tissue inhibitor of metalloproteinases expression. American journal of .... 2001.

26. Raghu G, Striker LJ, Hudson LD, Striker GE. Extracellular matrix in normal and fibrotic human lungs. Am Rev Respir Dis. 1985;131:281-9.

27. Leeming D, Alexandersen P, Karsdal M, Qvist P, Schaller S, Tanko L. An update on biomarkers of bone turnover and their utility in biomedical research and clinical practice. Eur J Clin Pharmacol. 2006;62:781-92.

28. Lee SH, Shim HS, Cho SH, Kim SY, Lee SK, Son JY, Jung JY, Kim EY, Lim JE, Lee KJ, Park BH, Kang YA, Kim YS, Kim SK, Chang J, Park MS. Prognostic factors for idiopathic pulmonary fibrosis: clinical, physiologic, pathologic, and molecular aspects. Sarcoidosis Vasc Diffuse Lung Dis. 2011;28:102-12.

29. du Bois R, Nathan S, Richeldi L, Schwarz M, Noble P. Idiopathic pulmonary fibrosis: lung function is a clinically meaningful endpoint for phase III trials. Am J Respir Crit Care Med. 2012;186:712-5.

30. Russell A-M, Adamali H, Molyneaux PL, Lukey PT, Marshall RP, Renzoni EA, Wells AU, Maher TM. Daily home spirometry: an effective tool for detecting progression in idiopathic pulmonary fibrosis. Am J Respir Crit Care Med. 2016;194:989-97.

31. Maher TM, Stowasser S, Nishioka Y, White ES, Cottin V, Noth I, Selman M, Blahova Z, Wachtlin D, Diefenbach C, Jenkins RG. Investigating the effects of nintedanib on biomarkers of extracellular matrix turnover in patients with IPF: design of the randomised placebo-controlled INMARK(R)trial. BMJ Open Respir Res. 2018;5:e000325.

32. Maher TM. PROFILEing idiopathic pulmonary fibrosis: rethinking biomarker discovery. Eur Respir Rev. 2013;22:148-52.

\section{Publisher's Note}

Springer Nature remains neutral with regard to jurisdictional claims in published maps and institutional affiliations.
Ready to submit your research? Choose BMC and benefit from:

- fast, convenient online submission

- thorough peer review by experienced researchers in your field

- rapid publication on acceptance

- support for research data, including large and complex data types

- gold Open Access which fosters wider collaboration and increased citations

- maximum visibility for your research: over $100 \mathrm{M}$ website views per year

At BMC, research is always in progress.

Learn more biomedcentral.com/submissions 